\title{
A Photometric Search for Circumstellar Shell Variability and Pre-Main Sequence Delta Scuti Variables
}

\author{
M. BREGER (Stony Brook) \\ (Read by J. HARDORP)
}

\begin{abstract}
A light variability study in NGC 2264 shows that about $25 \%$ of the pre-main sequence $A$ and $F$ stars show irregular short-period variability and/or brightness changes since 1953. There exists a good correlation between this variability and (other) shell indicators. The scatter in the pre-main sequence band in the color-magnitude diagram can be partially explained by shells and an age spread between 1 and $3 \times 10^{6}$ years is indicated. W 90, a star below the main sequence, has brightened by half a magnitude to $V=12.5$ since 1953. The corresponding color variation suggests a ratio of total to selective extinction of about 9 .

Two $\delta$ Scuti variables with periods of three hours were detected. Arguments for cluster membership are given. If cluster membership is confirmed, then they would be the first known cepheid-like pre-main sequence pulsators. The periods are short compared to similar post-main sequence stars. This could be interpreted as an unusually high mass or pulsation in a higher harmonic.

A large proportion of young stars hotter than the $T$ Tauri variables contracting towards the main sequence may be surrounded by gas and dust shells. Shell absorption would falsify the position of these stars in the observed color-magnitude diagram as well as the deduced stellar ages and star formation rates.

Shells are often unstable and the stars consequently show light variability over periods of hours as well as years. The very young cluster NGC 2264 is ideal for a light variability study since previously a very comprehensive photometric study was undertaken by WALKER (1956). In 15 years quite a few shell changes might be expected. Another interesting question is whether pre-main sequence stars inside the cepheid/ $\delta$ Scuti instability strip pulsate.

From 1968 to 1971, the two 36-inch telescopes at the Kitt Peak National Observatory were used with a photoelectric photometer to test 32 stars in and near the cepheid instability strip for variability. Rapid variability was investigated by observing four stars in rapid succession for a time of four or five hours. The techniques had previously been applied successfully to main sequence pulsators of small amplitudes of about 0.01 mag. (BREGER 1969, 1970). To monitor any night to night variations, the measurements were usually repeated on different nights with appropriate standards.

Out of $32 \mathrm{~A} / \mathrm{F}$ stars tested, 22 stars were constant to a few thousandths of a magnitude during several nights and showed a V magnitude unchanged from WALKER's (1956) magnitudes (within $\pm .03 \mathrm{mag}$ for photoelectric magnitudes).

Only two stars, W 2 (A 7III, IV) and W 20 (F 2III), showed regular or near regular light variability. Their light curves are shown in Figures 1 and 2. Their amplitudes of about .05 mag, short periods, shapes of light curves and slight beat phenomena are quite similar to those of typical $\delta$ Scuti variables. Ideally, one would wish to verify pulsation by matching the radial velocity, color and luminosity changes. However, at $10^{\text {th }}$ mag. exposure times would be too long to obtain reliable radial velocity measurements. Due to the similarity to post-main sequence variables, we conclude that $\mathrm{W}_{2}$ and $\mathrm{W} 20$ are pulsators.

Could the two variables actually be post-main sequence $\delta$ Scuti variables, i. e. nonmembers? Their short periods exclude the possibility of being background objects due to the periodluminosity relation for normal $\delta$ Scuti stars. Unfortunately, both pulsators are situated just outside the two regions in which proper motions and cluster membership were determined by VASILEVSKIS et al. (1965). On the other hand, W 20 shares the radial velocity and the ultraviolet excess of $\delta c_{1} \sim-0.2$ mag of the cluster's $F$ stars. Both variables are giants (rare amongst field stars) and their spectral class fits the cluster color-magnitude diagram. From the previous indirect arguments we conclude probable membership for W 2 and W 20.
\end{abstract}




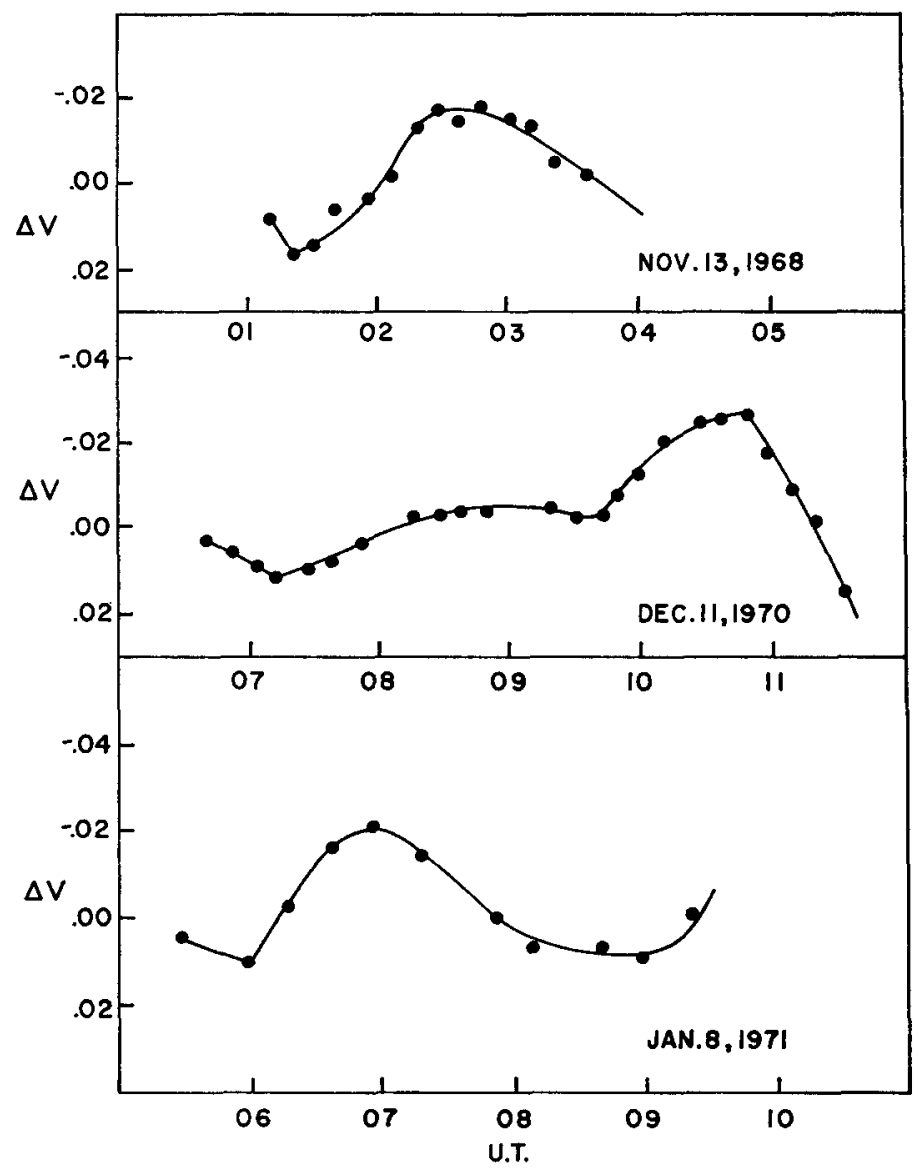

Fig. 1: Light curves of W 2 in NGC 2264, a $\delta$ Scuti variable with a period of 0.11 days.

From the basic equation of stellar structure and the pulsation criterion $P \sqrt{\bar{\varrho}} / \bar{\varrho} \odot=Q$, it can easily be shown that the pulsation periods $P$ of two stars of the same luminosity and temperature (and therefore radius) are related by

$$
\frac{\mathrm{P}_{1}}{\overline{\mathrm{P}}_{2}}=\frac{\mathrm{Q}_{1}}{\mathrm{Q}_{2}}\left(\frac{\mathrm{m}_{2}}{\mathrm{~m}_{1}}\right)^{1 / 2}
$$

where $\mathrm{m}$ represents the stellar mass. The pulsation constant is not quite constant and is a function of radius to mass ratio (e. g. see COGAN, 1970). The periods of the two new variables are only half of those expected for post-main sequence variables of the same luminosity and temperature. Inspection of equation (1) shows that the low periods could be explained by masses higher than those of post-main sequence stars or a low pre-main sequence $Q$ value which would require a small radius to mass ratio. Since the pre- and post-main sequence variables have the same radius (because of identical spectral types and positions in the color-magnitude diagram), a low $Q$ value also implies a large mass! The difference between classical pre- and post-main sequence evolutionary tracks accounts for less than $\sim 30 \%$ mass difference at that part of 


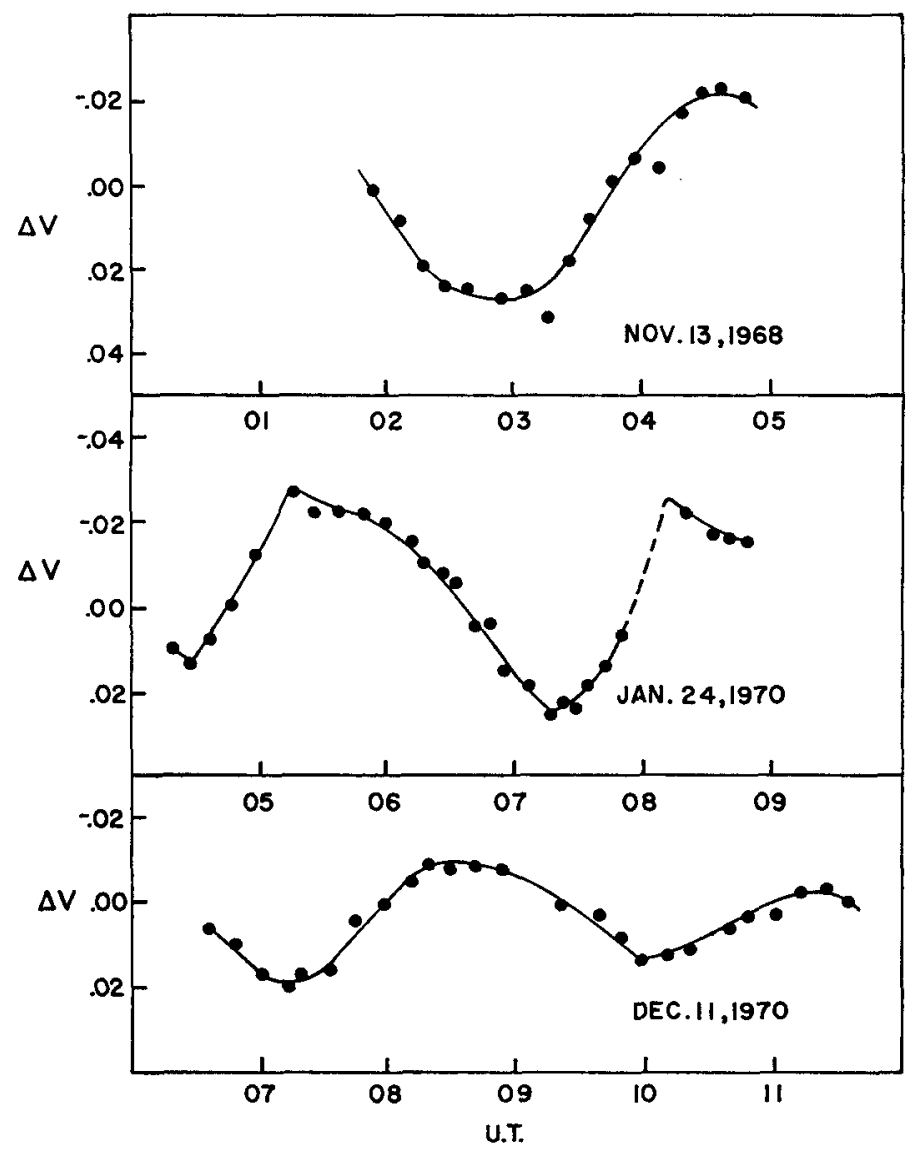

Fig. 2: Light curves of W 20 in NGC 2264, a $\delta$ Scuti variable with a period of 0.124 days.

the color-magnitude diagram. At constant $Q$ a mass of about $7 \pm 2$ solar masses is derived which means that $W_{2}$ and $W_{20}$ would join the already established main sequence at $B 5$, while the turn-on point occurs near AO. Finally, pulsation in the second or higher harmonic mode would also explain the short periods. We also note that should the two variables be surrounded by thick shells and be intrinsically brighter, the short periods found would be even more significant.

Only a few stars (W 90, W 108, W 189, W 224, and W 236) appear to have changed their brightness since 1953. The most spectacular variable is W90, an A 2 star, situated about one magnitude below the main sequence showing extremely strong shell characteristics such as an infrared excess in (V-L) of 6 mag. (STROM et al. 1971), high polarization, hydrogen line emission, light variation of several hundredths of a magnitude in a few hours as well as largeterm brightening from $V=13.2$ in 1954 to 12.5 in 1971 . The changes might be interpreted as expansion of the shell with a resulting drop in dust particle density in the line of sight. The ratio of total to selection absorption of the shell (or shell changes) may be calculated from the $\mathrm{V}$ and $(\mathrm{B}-\mathrm{V})$ changes. $\mathrm{A}$ value of $\mathrm{R}=\Delta \mathrm{V} / \Delta(\mathrm{B}-\mathrm{V}) \sim 9$ is obtained, much higher than the interstellar medium ratio of 3 . 
Also, W 90 and W 151 show irregular short-period variability, while the residuals of two more stars, W 165 and W 221, were regular enough to suggest possible variability of about .01 mag. Considering that three stars (including one variable) are proper motion non-members, only about $25 \%$ of the stars tested show light variability typical of shells. Figure 3 shows the non-random distribution of the variables in the color-magnitude diagram. The variables occur amongst the fainter $A / F$ stars at a given color as well as the cooler stars $(B-V \sim .6)$. It is attractive to speculate that the variability amongst the cooler stars is connected to the known shell variability in the even cooler T Tauri stars which start at $\mathrm{B}-\mathrm{V} \sim 0.9$.

We would like to suggest a relationship between light variability and shell thickness because of the following reasons: Shells are rarely stable and consequently shell stars often show light variability. Some examples are $\mathrm{T}$ Tauri stars, pre-main sequence $\mathrm{Ae}$ and $\mathrm{Be}$ stars in the field as well as "true" Be stars (e.g. FEINSTEIN's (1963) extensive work). Be and $T$ Tauri stars show much stronger emission lines than the average premain sequence $\mathrm{A} / \mathrm{F}$ star. However, large dust shell absorption need not be accompanied by very strong gas emission lines and changes in a thick dust shell should immediately be detected by a change in the visual magnitude. Our argument that the light variability is caused by shells is strengthened by the good correlation between light variability reported above and (other) shell characteristics (which are presently being investigated) such as large infrared excesses and polarization. We therefore feel that the light variability is caused by shells rather than intrinsic stellar variability.

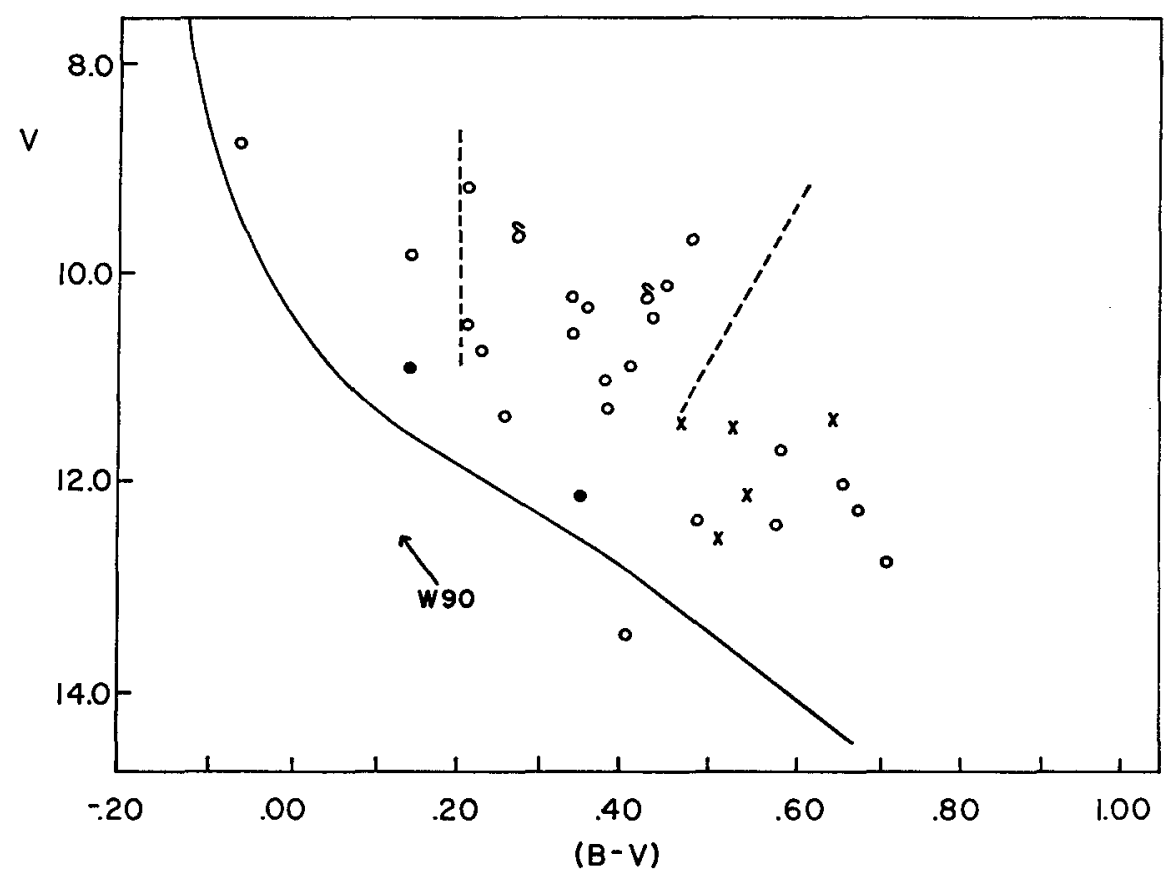

Fig. 3: Position of the variable stars in the color-magnitude diagram. Symbols are: 0 : constant, - : variability suspected, $X$ : irregular variability, $\delta: \delta$ Scuti variability. Solid and dashed lines are the ZAMS and borders of the instability, respectively, while the arrows denote the variation of $\mathrm{W} 90$ since 1954 . The diagram shows that the shell variables are generally faint and/or cool. 
The implications are that some, but not all of the scatter in the pre-main sequence is caused by circumstellar shells. Extensive shells would be present mainly in the fainter stars such as W 90. However, the observed lack of variability amongst the brighter A/F stars puts some limits on the presence of shells amongst these stars.

Let us assume for simplicity homologous shells with similar instabilities. Since the absorption in the visual $A_{v} \propto \mathrm{N}_{\mathrm{g}}$, the dust column density in the line of sight, the light variability of $\mathrm{W} 90$ would predict a column density about 30 times smaller in the nonvariable stars. Since W 90 (below the main sequence) has a visual absorption of two to three magnitudes, for the nonvariable stars a visual absorption of less than $0.2 \mathrm{mag}$ is predicted by possible shells. This would not affect the observed color-magnitude diagram. W 90 may, of course, be unusual. Nevertheless, in this interpretation the lack of variability excludes heavy visual absorption by a circumstellar shell so that the observed color-magnitude diagram of the brighter $\mathrm{A} / \mathrm{F}$ stars also represents the intrinsic magnitudes fairly well. Hence an age spread between 1 and $3 \times 10^{6}$ years is needed for these stars on the basis of classical evolutionary tracks.

$$
\text { Ref e rences: }
$$

BREGER, M., 1969, Ap. J., Suppl., 19, 79.

BREGER, M., 1970, A p. J., 162, 597.

COGAN, B. C., 1970, Ap. J., 162, 139.

FEINSTEIN, A., 1968, Zs. f. Ap., 68, 29.

STROM, K. M., STROM, S. E., and YOST, J., 1971, Ap. J., 165, 479.

VASILEVSKIS, S., SANDERS, W. L., and BÄLZ Jr., A.G.A., 196.5, Astron. J., 70, 797.

WALKER, M. F., 1956, Ap. J. Suppl., 2, 365.

\section{Discussion to the paper of BREGER}

SAHADE: Do you mean to imply that all $\delta$ Sct stars are pre-main sequence stars?

HARDORP: Not at all.

SAHADE: Then, perhaps a different designation should be used to distinguish the 2 groups. KIPPENHAHN: We have seen light curves which to me look rather irregular. Why should one assume that they are pulsating variables of $\delta$ Sct type?

PERCY: The two variables found by BREGER in NGC 2264 have periods and light curves similar to the many post-main sequence variables discovered by BREGER and for which good period-luminosity and period-colour relations hold and which are therefore periodic pulsating variables, rather than irregular variables.

WALKER: Are reliable spectral types available for all of the stars observed in this program? Among the fainter stars, one finds that unlike the early object studied by STROM, et al., the location of the star in the C-M diagram tends to be shifted primarily in the $B-V$ rather than the $V$ direction, owing to the occurrence in these stars of a blue continuum. It may be that this type of effect could occur in some of the fainter stars in the sample discussed here.

HARDORP: Since radial velocities have been determined for most of the stars, it should be no problem to obtain spectral types of the stars from the same plates, if this has not already been done.

AIZENMAN: The $\delta$ Sct stars have masses of $2.0 \mathrm{M}_{\odot}$. Does the $7 \mathrm{M}_{\odot}$ star come down to this region in the pre-main sequence contraction phase?

HARDORP: No. 